\title{
Peptide nanomaterials as future antimicrobial technologies
}

\author{
Garry Laverty ${ }^{*}, 1$ \\ ${ }^{1}$ Biofunctional Nanomaterials Group, School of Pharmacy, Queen's University Belfast, Belfast, Co Antrim BT9 7BL, Northern Ireland \\ * Author for correspondence: garry.laverty@qub.ac.uk
}

\begin{abstract}
"Our research groups are currently assessing if nanotubes have the added ability to extend the spectrum of activity of current Gram-positive selective antibiotics and deliver them across the lipopolysaccharide outer membrane barrier, providing synergistic action."”
\end{abstract}

First draft submitted: 20 September 2017; Accepted for publication: 26 September 2017; Published online: 4 December 2017

Keywords: biomaterials $\bullet$ drug delivery $\bullet$ infection $\bullet$ peptide $\bullet$ peptidomimetics

\section{Peptides in medicine}

Peptides have long been heralded as promising molecules for the treatment and management of a variety of disease states including cancer, diabetes and inflammatory disease [1]. They represent the interface between naturally occurring biologics and chemically synthesized active pharmaceutical ingredients, offering high selectivity for molecular targets, leading to increased therapeutic efficacy and lower capacity for side effects. Their widespread implementation throughout medicine has been limited by their relatively expensive cost of manufacture and poor bioavailability. Despite these challenges, researchers, clinicians and the pharmaceutical industry continue to work together in an attempt to clinically translate peptides of therapeutic value. Issues relating to ease and cost of synthesis can be remedied by creating ultrashort peptide pharmacophores, composed of seven or less amino acids, which retain pharmacological properties of larger peptides and proteins. Short peptides are more attractive to the pharmaceutical industry as it is cheaper to scale up their manufacture and they are more likely to be clinically translated for the benefit of patients. The area of peptidomimetics, including $\beta$-peptides [2], D-enantiomeric variants [3] and peptoids [4], has resulted in a wealth of unnatural peptide-like structures which demonstrate improved stability profiles. Their success is exemplified by orally active angiotensin-converting enzyme inhibitors, for example, captopril, widely prescribed for the treatment of hypertension and congestive heart failure since the early 1980s [5].

\section{Peptides as antimicrobials}

The potential of peptides in antimicrobial therapy stems from their role as mediators of the innate immune response, serving as important weapons in nature and their use is increasing in medicine for combating infection and controlling the host response to pathogens [6]. Their promise lies in not only their ability to provide direct antimicrobial action via a variety of extracellular and intracellular targets but also their potential to harness the body's own defenses, utilizing the ubiquitous properties peptides possess throughout nature, to prevent infection. This is demonstrated by the use of peptides as immune adjuvants in vaccine development, replacing components with questionable safety such as aluminium [7]. Traditional antimicrobials are susceptible to resistance development due to the targeting of specific microbial receptors or biomolecular mechanisms [8]. There is an urgent need to develop the next generation of antimicrobial therapies, which act using several mechanisms, to limit induction of drug resistance and address the challenge of multidrug resistance. Serious concerns exist regarding increased antimicrobial resistance and the relative lack of new antimicrobials in development [9]. Peptides are not completely devoid of resistance development, however, with recent reports of colistin's reduced efficacy in China [10] and the elucidation of the two-component systems PhoP/PhoQ and PmrA/PmrB pathways which result in cationic modification of the membrane bound anchor lipid A by UDP-4-amino-4-deoxy-L-arabinose, leading to increased bacterial resistance to cationic antimicrobial peptides and polymyxins [6,11]. Despite such reports, peptides still serve as promising molecules to fill the current void in antimicrobial drug development. Due to toxicity and

Future Medicine 
stability concerns, their marketed indications have been limited to the treatment of skin and tissue infections, although a variety of new routes of administration are forthcoming as exemplified by daptomycin, licensed in the USA as an intravenous therapy for right-sided endocarditis [12]. Researchers continue to explore a variety of new peptide templates for improved antimicrobial efficacy, reduced toxicity and greater microbial specificity, harnessing the power of molecular modeling and structure-activity relationships to guide the design of unique amino acid sequences [13]. An increasingly exciting field of research involves the development of peptide-based nanomaterials which can be designed from the 'bottom-up' to display optimal functional and pharmacological characteristics for their intended use.

\section{Peptides as nanomaterial therapeutics}

Just as peptides can be tailored to demonstrate antimicrobial characteristics, their structure can be modified to enable them to assemble spontaneously into highly defined nanostructures. These self-assembling peptides are composed of amino acid building blocks which organize, via intermolecular interactions, into nanomaterial structures in response to physiological stimuli including: $\mathrm{pH}$, temperature, ionic strength and the presence of specific enzymes [14]. By modifying the amino acid sequence, we can control whether these peptides assemble into hydrogels composed of nanofibers or peptide nanotube structures. Their responsiveness to changing physiological stimuli makes them promising platforms throughout the drug delivery and biomaterial fields. The properties that dictate a peptide's ability to self-assemble are very similar to the principles that govern the activity of antimicrobial peptides, most significant of which is the hydrophobic:hydrophilic charge balance of the peptide primary sequence [14]. As a result, researchers are able to control not only the process of self-assembly in response to stimuli, but also pharmacological properties such as antimicrobial activity. In this way, biofunctional nanomaterials can be designed and developed for specific functional requirements. These platforms have huge potential within infection treatment and prevention. Peptides are ideal materials for medical applications as they can be designed to demonstrate inherent biocompatibility, antimicrobial activity, biodegradability and possess numerous chemical groups which can be utilized to tailor functional properties (e.g., mechanical strength, stability, hydrogel formation).

They have many advantages over current synthetic materials used in healthcare. Peptides possess vast chemical versatility due to the varying nature of their amino acid R-group. This is proven by how they are harnessed throughout nature. They can be utilized to create materials with very specific functionalities and with the potential to attach a variety of molecules including drugs [15]. As amino acids represent the building blocks of peptide, proteins and tissues, they exist throughout the body possessing enhanced biocompatibility compared with synthetic-based polymeric and nanomaterial systems. The amino acid sequence can also be altered to tailor biodegradation to specific requirements and release conjugated drug molecules for improved efficacy. Biologically derived peptides are proteins, from animals and plants, which have been studied extensively for use as medical materials (e.g., collagen, silk) $[16,17]$. However their use is limited as they require expensive post modification to both purify and tailor mechanical and biological properties to their clinical purpose.

\section{Future peptide nanomaterial technologies}

Self-assembling peptide materials demonstrate significant potential for clinical translation as evidenced by the example of octreotide - which has been recently granted regulatory approval as a therapy for acromegaly [1]. Both peptide hydrogel and nanotube platforms have a variety of potential clinical applications in the antimicrobial field. Their most obvious use is as a hydrogel wound therapy, given that peptides form the building blocks of tissues and skin. Peptides can be manufactured within the laboratory to reproduce the unique mechanical and biological properties of natural tissue, with the potential to provide both antimicrobial and anti-inflammatory action [15]. Peptide hydrogels could represent the next generation of medical device coatings, triggered to respond to infection development via pathogenic stimuli (e.g., specific bacterial enzymes) thereby providing prolonged protection from bacterial attachment and biofilm formation $[18,19]$. An easy-to-synthesize low-molecular-weight peptide has the potential to widely be exploited as immune adjuvants for vaccines, 3D cell culture, cell and bioactive molecule (e.g., genes) delivery, bioimaging agents and diagnostics. Peptide nanotube platforms have huge potential as drug delivery platforms for transversing biological barriers, for example, the outer membrane of Gram-negative bacteria, mimicking the value of their carbon counterparts in terms of therapeutic applications but with improved chemical functionality for drug conjugation and reduced toxicity concerns [20]. Their value may lie in encapsulating and extending the spectrum of activity of licensed Gram-positive selective antibiotics for delivery across the outer membrane of Gram-negative bacteria including Pseudomonas aeruginosa, Escherichia coli, Klebsiella pneumoniae and 
Acinetobacter baumannii. This membrane is a major physical barrier to the entry of antibiotics in Gram-negative infection and a significant contributor to antimicrobial resistance. There is a serious lack of available, effective, narrow spectrum Gram-negative selective antibiotics in development. Our research groups are currently assessing if nanotubes have the added ability to extend the spectrum of activity of current Gram-positive selective antibiotics and deliver them across the lipopolysaccharide outer membrane barrier, providing synergistic action. Using existing, licensed antibiotics should prove advantageous in limiting the regulatory barriers and time to clinical translation, an important consideration in the fight against the increasing menace of antimicrobial resistance.

\section{Financial \& competing interests disclosure}

This work was funded by Royal Society (IE160988) and Wellcome Trust (207618/Z/17/Z) research grants for G Laverty. The author has no other relevant affiliations or financial involvement with any organization or entity with a financial interest in or financial conflict with the subject matter or materials discussed in the manuscript apart from those disclosed.

No writing assistance was utilized in the production of this manuscript.

\section{References}

1. Rafferty J, Nagaraj H, McCloskey AP et al. Peptide therapeutics and the pharmaceutical industry: barriers encountered translating from the laboratory to patients. Curr. Med. Chem. 23(37), 4231-4259 (2016).

2. Cabrele C, Martinek TA, Reiser O, Berlicki L. Peptides containing beta-amino acid patterns: challenges and successes in medicinal chemistry. J. Med. Chem. 57(23), 9718-9739 (2014).

3. Feng Z, Xu B. Inspiration from the mirror: D-amino acid containing peptides in biomedical approaches. Biomol. Concepts 7(3), 179-187 (2016).

4. Wu Z, Tan M, Chen X, Yang Z, Wang L. Molecular hydrogelators of peptoid-peptide conjugates with superior stability against enzyme digestion. Nanoscale 4(12), 3644-3646 (2012).

5. De Leo F, Panarese S, Gallerani R, Ceci LR. Angiotensin converting enzyme (ACE) inhibitory peptides: production and implementation of functional food. Curr. Pharm. Des. 15(31), 3622-3643 (2009).

6. Laverty G, Gorman SP, Gilmore BF. The potential of antimicrobial peptides as biocides. Int. J. Mol. Sci. 12(10), 6566-6596 (2011).

7. Wen Y, Collier JH. Supramolecular peptide vaccines: tuning adaptive immunity. Curr. Opin. Immunol. 35, 73-79 (2015).

8. Munita JM, Arias CA. Mechanisms of antibiotic resistance. Microbiol. Spectr. 4(2), doi:10.1128/microbiolspec.VMBF-0016-2015 (2016).

9. World Health Organization. Antimicrobial resistance: global report on surveillance 2014 (2014). www.who.int/drugresistance/documents/surveillancereport/en/

10. Liu YY, Wang Y, Walsh TR et al. Emergence of plasmid-mediated colistin resistance mechanism MCR-1 in animals and human beings in China: a microbiological and molecular biological study. Lancet Infect. Dis. 16(2), 161-168 (2016).

11. Laverty G, Gorman SP, Gilmore BF. Biomolecular mechanisms of Pseudomonas aeruginosa and Escherichia coli biofilm formation. Pathogens 3(3), 596-632 (2014).

12. Gonzalez-Ruiz A, Seaton RA, Hamed K. Daptomycin: an evidence-based review of its role in the treatment of Gram-positive infections. Infect. Drug Resist. 9, 47-58 (2016).

13. Abraham T, Prenner EJ, Lewis RN et al. Structure-activity relationships of the antimicrobial peptide gramicidin $S$ and its analogs: aqueous solubility, self-association, conformation, antimicrobial activity and interaction with model lipid membranes. Biochim. Biophys. Acta 1838(5), 1420-1429 (2014).

14. McCloskey AP, Gilmore BF, Laverty G. Evolution of antimicrobial peptides to self-assembled peptides for biomaterial applications. Pathogens 3(4), 791-821 (2014).

15. McCloskey AP, Gilmore SM, Zhou J et al. Self-assembling ultrashort NSAID-peptide nanosponges: multifunctional antimicrobial and anti-inflammatory materials. RSC Adv. 6, 114738-114749 (2016).

16. Huang W, Rollett A, Kaplan DL. Silk-elastin-like protein biomaterials for the controlled delivery of therapeutics. Expert Opin. Drug Deliv. 12(5), 779-791 (2015).

17. Jao D, Xue Y, Medina J, Hu X. Protein-based drug-delivery materials. Materials (Basel) 10(5), E517 (2017).

18. Hughes M, Debnath S, Knapp CW, Ulijn RV. Antimicrobial properties of enzymatically triggered self-assembling aromatic peptide amphiphiles. Biomater. Sci. 11, 1138-1142 (2013).

19. Laverty G, McCloskey AP, Gilmore BF, Jones DS, Zhou J, Xu B. Ultrashort cationic naphthalene-derived self-assembled peptides as antimicrobial nanomaterials. Biomacromolecules 15(9), 3429-3439 (2014).

20. Huwaitat R, McCloskey AP, Gilmore BF, Laverty G. Potential strategies for the eradication of multidrug-resistant Gram-negative bacterial infections. Future Microbiol. 11(7), 955-972 (2016). 
